Elect. Comm. in Probab. 15 (2010), 281-10

\title{
ON THE DISTRIBUTION OF THE BROWNIAN MOTION PROCESS ON ITS WAY TO HITTING ZERO
}

\section{KONSTANTIN BOROVKOV ${ }^{1}$}

Department of Maths \& Stats, The University of Melbourne, Parkville 3010, Australia

email: borovkov@unimelb.edu.au

Submitted 14 Dec 2009, accepted in final form 15 Jun 2010

AMS 2000 Subject classification: 60J65

Keywords: Brownian motion, hitting time, Brownian meander, Bessel bridge

Abstract

We present functional versions of recent results on the univariate distributions of the process $V_{x, u}=x+W_{u \tau(x)}, 0 \leq u \leq 1$, where $W_{\bullet}$ is the standard Brownian motion process, $x>0$ and $\tau(x)=\inf \left\{t>0: W_{t}=-x\right\}$.

Let $\left\{W_{t}\right\}_{t \geq 0}$ be the standard univariate Brownian motion process and, for $x>0$,

$$
W_{x, t}:=x+W_{t}, \quad t \geq 0, \quad \tau(x):=\inf \left\{t>0: W_{x, t}=0\right\} .
$$

As is well known, $\tau(x)$ is a proper random variable with density

$$
p_{x}(t)=\frac{x e^{-x^{2} / 2 t}}{\sqrt{2 \pi t^{3}}}, \quad t>0
$$

so one can introduce

$$
V_{x, u}:=W_{x, u \tau(x)}, \quad 0 \leq u \leq 1 .
$$

These random variables were studied in the recent paper [5], where it was shown (Theorem 1.1) that, for any fixed $u \in(0,1), V_{x, u}$ has density

$$
\begin{aligned}
p_{x, u}(y) & :=\frac{d}{d y} \mathbf{P}\left(V_{x, u} \leq y\right) & \\
& =\frac{4 \sqrt{u(1-u)} x y^{2}}{\pi\left(u y^{2}+(1-u)(y-x)^{2}\right)\left(u y^{2}+(1-u)(y+x)^{2}\right)}, & y>0, \\
& \sim \frac{4 x \sqrt{u(1-u)}}{\pi y^{2}} & \text { as } y \rightarrow \infty
\end{aligned}
$$

\footnotetext{
${ }^{1}$ RESEARCH SUPPORTED BY ARC DISCOVERY GRANT DP0880693
} 
(here and in what follows, $a \sim b$ means that $a / b \rightarrow 1$ ). Representation (2) implies, in particular, that, for any fixed $u \in[0,1]$, one has

$$
V_{x, u} \stackrel{d}{=} x V_{1, u}
$$

Using a direct tedious calculation, it was also demonstrated in Section 3 of [5] that, for a fixed $u \in(0,1)$, the density $p_{x, u}$ coincides with that of a "scaled Brownian excursion at the corresponding time, averaged over its length". The mathematical formulation of that result was given by formula (3.3) in [5] that can be rewritten as follows. Let $\left\{R_{x, t}^{T}\right\}_{t \leq T}$ be a three-dimensional Bessel bridge of length $T$ pinned at $x$ at time $t=0$ and at 0 at time $t=T$, which is independent of our process $W_{x, \bullet}$ (and hence of $\tau(x)$ ). Recall that one can represent the process as

$$
R_{x, t}^{T}=\left\|W_{x, t}^{(3)}-t T^{-1} W_{x, T}^{(3)}\right\|, \quad 0 \leq t \leq T,
$$

where

$$
W_{x, t}^{(3)}=(x, 0,0)+W_{t}^{(3)}, \quad t \geq 0,
$$

$W_{\bullet}^{(3)}$ being a standard three-dimensional Brownian motion process and $\|\cdot\|$ the Euclidean norm in $\mathbb{R}^{3}$. The above-mentioned formula from [5] is equivalent to the assertion that, for any fixed $u \in[0,1]$, one has

$$
V_{x, u} \stackrel{d}{=} R_{x, u \tau(x)}^{\tau(x)}
$$

Note that $R_{x, \bullet}^{T}$ is not exactly an excursion (an excursion returns to the same point where it started) but, rather, a time-reversed Brownian meander (see e.g. p.63 in [2]), and that on the right-hand side of (7) it is averaged not over its length, but rather of that of an independent version of $W_{x, \bullet}$ on its way to hitting zero.

Observe also that, due to the self-similarity of the Brownian motion process, representation (5)(6) implies that

$$
R_{x, \bullet T}^{T} \stackrel{d}{=} T^{1 / 2} R_{T^{-1 / 2} x, \bullet}
$$

(as processes in $C[0,1]$ ), where we put $R_{x, t}:=R_{x, t}^{1}$.

The main aim of the present note is to give simple proofs to functional versions of (4) and (7) (that had "remained elusive", as was noted in [5]).

Theorem 1. For any $x>0$,

$$
\left\{V_{x, u}\right\}_{u \leq 1} \stackrel{d}{=}\left\{x V_{1, u}\right\}_{u \leq 1} .
$$

Furthermore, there exists a regular version of the conditional distribution of $V_{1, \bullet}$ in $C[0,1]$ given $\tau(1)=T$ that coincides with the law of $T^{1 / 2} R_{T^{-1 / 2}, \bullet}$, and therefore, if $\tau \stackrel{d}{=} \tau(1)$ is independent of the Brownian motion process from representation (5)-(6), then one has

$$
\left\{V_{1, u}\right\}_{u \leq 1} \stackrel{d}{=}\left\{\tau^{1 / 2} R_{\tau^{-1 / 2}, u}\right\}_{u \leq 1}
$$

Proof of Theorem 1. First we observe that

$$
W_{x, t}=x\left(1+x^{-1} W_{t}\right)=x \widetilde{W}_{1, t x^{-2}}, \quad t \geq 0
$$

where $\widetilde{W}_{1}$, is a Brownian motion process starting at 1 . All quantities related to this process we will label with tilde. As $\tau(x)$ is the first time the LHS of (10) turns into zero, we see that $\tilde{\tau}(1)=\tau(x) x^{-2}$. Therefore

$$
V_{x, u}=W_{x, u \tau(x)}=x \widetilde{W}_{1, u \tau(1)}=x \widetilde{V}_{1, u}, \quad u \in[0,1]
$$


which proves (8). So from now on, we can assume without loss of generality that $x=1$.

Next let, for some functions $f_{j} \in C[0,1]$ and numbers $r_{j}>0, j=1,2, \ldots, n$,

$$
A:=\bigcap_{j \leq n}\left\{f \in C[0,1]:\left\|f-f_{j}\right\|<r_{j}\right\}
$$

be a finite intersection of open balls in $C[0,1](\|\cdot\|$ stands for the uniform norm). For $T, h, \delta>0$, put

$$
A_{T}:=\{f(\bullet / T): f \in A\} \subset C[0, T], \quad \varepsilon(\delta):=\max _{j \leq n} \omega_{f_{j}}(\delta),
$$

where $\omega_{f}(\delta):=\sup _{0 \leq s<t \leq s+\delta \leq 1}|f(s)-f(t)|$ is the continuity modulus of the function $f$. Finally, we denote by $A_{T}^{\varepsilon(h / T)}$ the $\varepsilon(h / T)$-neighbourhood of $A_{T}$ (in the uniform topology on $C[0, T]$ ) and introduce the event

$$
B_{T, h}:=\left\{\left\{W_{1, t}\right\}_{t \in[0, T]} \in A_{T}^{\varepsilon(h / T)}\right\} .
$$

Now, employing notation $\check{X}_{t}:=\inf _{s \leq t} X_{s}$, the Markov property and the well-know relations

$$
\mathbf{P}\left(\check{W}_{h}<0 \mid W_{0}=y\right)=2 \bar{\Phi}\left(y h^{-1 / 2}\right), \quad \mathbf{P}\left(\check{W}_{1, T}>0 \mid W_{1, T}=y\right)=1-e^{-2 y / T}, \quad y>0,
$$

where $\bar{\Phi}=1-\Phi, \Phi$ being the standard normal distribution function, we have

$$
\begin{aligned}
\mathbf{P} & \left.V_{1, \bullet} \in A, \tau(1) \in(T, T+h)\right) \leq \mathbf{P}\left(B_{T, h}, \tau(1) \in(T, T+h)\right) \\
& =\int_{0}^{\infty} \mathbf{P}\left(B_{T, h}, \tau(1) \in(T, T+h) \mid W_{1, T}=y\right) \mathbf{P}\left(W_{1, T} \in d y\right) \\
& =\int_{0}^{\infty} \mathbf{P}\left(B_{T, h}, \check{W}_{1, T}>0, \check{W}_{1, T+h}<0 \mid W_{1, T}=y\right) \mathbf{P}\left(W_{1, T} \in d y\right) \\
& =\int_{0}^{\infty} \mathbf{P}\left(B_{T, h}, \check{W}_{1, T}>0 \mid W_{1, T}=y\right) \mathbf{P}\left(\min _{t \in[T, T+h]} W_{1, t}<0 \mid W_{1, T}=y\right) \mathbf{P}\left(W_{1, T} \in d y\right) \\
& =\int_{0}^{\infty} \mathbf{P}\left(B_{T, h} \mid \check{W}_{1, T}>0, W_{1, T}=y\right) \mathbf{P}\left(\check{W}_{1, T}>0 \mid W_{1, T}=y\right) 2 \bar{\Phi}\left(y h^{-1 / 2}\right) \mathbf{P}\left(W_{1, T} \in d y\right) \\
& =2 \int_{0}^{\infty} \mathbf{P}\left(B_{T, h} \mid \check{W}_{1, T}>0, W_{1, T}=y\right)\left(1-e^{-2 y / T}\right) \bar{\Phi}\left(y h^{-1 / 2}\right) \mathbf{P}\left(W_{1, T} \in d y\right) \\
& =(4+o(1)) h^{1 / 2} \int_{0}^{h^{1 / 4}} \mathbf{P}\left(B_{T, h} \mid \check{W}_{1, T}>0, W_{1, T}=y\right) g_{T}\left(y h^{-1 / 2}\right) d y+o(h)
\end{aligned}
$$

as $h \downarrow 0$, where

$$
g_{T}(u)=\frac{1}{\sqrt{2 \pi}} u T^{-3 / 2} e^{-1 /(2 T)} \bar{\Phi}(u), \quad u>0,
$$

and we used the well-known Mills ratio asymptotics $\bar{\Phi}(u) \sim(2 \pi)^{-1 / 2} u^{-1} e^{-u^{2} / 2}, u \rightarrow \infty$, to infer that $\int_{h^{1 / 4}}^{\infty}=o(h)$

Next we will show that the probability in the last integrand in (11) converges to the respective probability for the Brownian meander process as $y \downarrow 0$.

Recall that the Brownian meander process $\left\{W_{s}^{\oplus}\right\}_{s \leq 1}$ can be defined as follows (see e.g. [4] or p.64 in [2]): letting $\zeta:=\sup \left\{t \leq 1: W_{t}=0\right\}$ be the last zero of the Brownian motion in [0,1], we set

$$
W_{s}^{\oplus}:=(1-\zeta)^{-1 / 2}\left|W_{\zeta+(1-\zeta) s}\right|, \quad 0 \leq s \leq 1 .
$$


This is a continuous nonhomogeneous Markov process whose transition density can be found e.g. in [4] (relations (1.1) and (1.2)). It is known that the conditional version of the process pinned at $x>0$ at time $s=1$ coincides in distribution with the three-dimensional Bessel process starting at zero and also pinned at $x$ at time $s=1$ (see e.g. p.64 in [2]), which can be written as

$$
\mathscr{L}\left(\left\{W_{s}^{\oplus}\right\}_{s \leq 1} \mid W_{1}^{\oplus}=x\right)=\mathscr{L}\left(\left\{\left\|W_{s}^{(3)}\right\|\right\}_{s \leq 1} \mid\left\|W_{1}^{(3)}\right\|=x\right)
$$

(here and in what follows, $\mathscr{L}(X \mid C)$ denotes the conditional distribution of the random element $X$ in the respective measureable space given condition $C, \mathscr{L}(X)$ stands for the unconditional distribution of $X$ ). It is not hard to deduce from here, the spherical symmetry of the Brownian motion process $W_{\bullet}^{(3)}$ and representation (5)-(6) above that

$$
\mathscr{L}\left(\left\{W_{s}^{\oplus}\right\}_{s \leq 1} \mid W_{1}^{\oplus}=x\right)=\mathscr{L}\left(\left\{\left\|W_{x, 1-s}^{(3)}-(1-s) W_{x, 1}^{(3)}\right\|\right\}_{s \leq 1}\right)=\mathscr{L}\left(\left\{R_{x, 1-s}\right\}_{s \leq 1}\right) .
$$

An alternative insightful interpretation of the Brownian meander is given by the fact that its distribution (in $C[0,1]$ ) coincides with the weak limit of conditional distributions of $W_{\bullet}$ conditioned to stay above $-\varepsilon \uparrow 0$ :

$$
\mathscr{L}\left(\left\{W_{s}^{\oplus}\right\}_{s \leq 1}\right)=\mathrm{w}-\lim _{\varepsilon \downarrow 0} \mathscr{L}\left(\left\{W_{s}\right\}_{s \leq 1} \mid \breve{W}_{1}>-\varepsilon\right)
$$

(Theorem (2.1) in [4]; w-lim stands for the limit in weak topology). A conditional version of a result of this type is used in the calculation displayed in (13) below.

Now return to the probability in the integrand in the last line in (11) and recall the well-known property of Brownian bridges that conditioning a Brownian motion on its arrival at a point $y \neq 0$ at time $T$ is equivalent to conditioning on its arrival to zero at that time and then adding the deterministic linear trend component $y t / T$. This implies that, for any $\varepsilon \geq \varepsilon(h / T)$,

$$
\begin{aligned}
\mathbf{P} & \left(B_{T, h} \mid \breve{W}_{1, T}>0, W_{1, T}=y\right) \\
& =\mathbf{P}\left(\left\{W_{1, t}+y t T^{-1}\right\}_{t \leq T} \in A_{T}^{\varepsilon(h / T)} \mid W_{1, T}=0 ; W_{1, s}>-y s T^{-1}, s \in[0, T]\right) \\
& =\mathbf{P}\left(\left\{W_{T-t}+y t T^{-1}\right\}_{t \leq T} \in A_{T}^{\varepsilon(h / T)} \mid W_{T}=1 ; W_{s}>-y(T-s) T^{-1}, s \in[0, T]\right) \\
& =\mathbf{P}\left(\left\{T^{1 / 2} W_{1-v}+y v\right\}_{v \leq 1} \in A^{\varepsilon(h / T)} \mid W_{1}=T^{-1 / 2} ; W_{v}>-y T^{-1 / 2}(1-v), v \in[0,1]\right) \\
& \leq \mathbf{P}\left(\left\{T^{1 / 2} W_{1-v}+y v\right\}_{v \leq 1} \in A^{\varepsilon} \mid W_{1}=T^{-1 / 2} ; W_{v}>-y T^{-1 / 2}(1-v), v \in[0,1]\right) \\
& \rightarrow \mathbf{P}\left(\left\{T^{1 / 2} W_{1-v}^{\oplus}\right\}_{v \leq 1} \in A^{\varepsilon} \mid W_{1}^{\oplus}=T^{-1 / 2}\right) \\
& =\mathbf{P}\left(\left\{T^{1 / 2} R_{T^{-1 / 2}, s}\right\}_{s \leq 1} \in A^{\varepsilon}\right)
\end{aligned}
$$

as $y \downarrow 0$, where the second last relation follows from the weak convergence established in Theorem 6 in [3] (as it is obvious that $A^{\varepsilon}$ has null boundary w.r.t. the limiting distribution) and the last one follows from $(12)$.

Since $\varepsilon(h / T) \rightarrow 0$ as $h \downarrow 0$, and $A$ has a null boundary under $\mathscr{L}\left(\left\{T^{1 / 2} R_{T^{-1 / 2}, s}\right\}_{s \leq 1}\right)$, we conclude from (11) (changing there the variables: $u=y h^{-1 / 2}$ ) that

$$
\begin{aligned}
\underset{h \downarrow 0}{\limsup } & \frac{1}{h} \mathbf{P}\left(V_{1, \bullet} \in A, \tau(1) \in(T, T+h)\right) \\
& \leq \underset{h \downarrow 0}{\limsup } 4 \mathbf{P}\left(T^{1 / 2} R_{T^{-1 / 2}, \bullet} \in A\right) \int_{0}^{h^{-1 / 4}} g_{T}(u) d u \\
& =\mathbf{P}\left(T^{1 / 2} R_{T^{-1 / 2}}, \in A\right) p_{1}(T),
\end{aligned}
$$


owing to $\int_{0}^{\infty} u \bar{\Phi}(u) d u=\frac{1}{4}$ and (1).

As the same argument as employed in (13) and (14) will also work for the complement of $A$, we obtain that

$$
\mathbf{P}\left(V_{1, \bullet} \in A, \tau(1) \in(T, T+h)\right) \sim \mathbf{P}\left(T^{1 / 2} R_{T^{-1 / 2}}, \in A\right) p_{1}(T) h \quad \text { as } \quad h \downarrow 0 .
$$

This relation implies that, for any fixed $0<T_{1}<T_{2}<\infty$,

$$
\mathbf{P}\left(V_{1, \bullet} \in A, \tau(1) \in\left(T_{1}, T_{2}\right)\right)=\int_{T_{1}}^{T_{2}} \mathbf{P}\left(T^{1 / 2} R_{T^{-1 / 2}, \bullet} \in A\right) p_{1}(T) d T .
$$

Since intersections of finite collections of open balls form determining classes in separable spaces (see e.g. Section I.2 in [1]), this completes the proof of the theorem.

Acknowledgment. The author is grateful to the referee whose valuable comments helped to improve the exposition of the paper.

\section{References}

[1] Patrick Billingsley. Convergence of Probability Measures. Wiley, New York, second edition, 1999. MR1700749

[2] Andrei N. Borodin and Paavo Salminen. Handbook of Brownian motion - Facts and Formulae. Birkhäuser Verlag, Basel, second edition, 2002. MR1912205

[3] Konstantin Borovkov and Anrew N. Downes. On boundary crossing probabilities for diffusion processes. Stoch. Proc. Appl. 120(2): 105-129, 2010. MR2576883

[4] Richard T. Durrett, Donald L. Iglehart and Douglas R. Miller. Weak convergence to Brownian meander and Brownian excursion. Ann. Probab. 5(1):117-129, 1977. MR0436353

[5] Pavel Chigansky and Fima C. Klebaner. Distribution of the Brownian motion on its way to hitting zero. Electr. Comm. Probab. 13:641-648, 2008. MR2466191 